\title{
CARACTERIZAÇÃO DO DESENVOLVIMENTO DA ALIMENTAÇÃO EM CRIANÇAS DE 6 AOS 24 MESES DE IDADE DO MUNICÍPIO DE CANOAS/RS
}

\author{
Characterization of feeding development \\ in 6 to 24 month-old children in Canoas/RS
}

\author{
Aline e Silva Carneiro ${ }^{(1)}$, Susana Elena Delgado ${ }^{(2)}$, Silvana Maria Brescovici ${ }^{(3)}$
}

\begin{abstract}
RESUMO
Objetivos: caracterizar a alimentação de crianças na faixa etária entre seis e 24 meses verificando a época de introdução, tipos de alimentos e texturas oferecidas conforme a faixa etária, bem como, verificar hábitos orais e constatar quem orientou os pais sobre alimentação. Métodos: a amostra foi composta por 100 crianças dos seis aos 24 meses, que frequentaram duas unidades básicas de saúde, em Canoas, RS. Foram realizadas entrevistas com os responsáveis. Resultados: constatouse que $98 \%(\mathrm{~N}=98)$ das crianças foram amamentadas exclusivamente, porém com duração média de dois meses e oito dias. O oferecimento de líquidos apresentou introdução precoce de chá com mediana de início aos 60 dias; $92 \%(\mathrm{~N}=92)$ das crianças usaram mamadeira e destas em $47 \%(\mathrm{~N}=47)$ aumentaram o furo. A chupeta era usada por $60 \%(\mathrm{~N}=60)$ da amostra e teve associação com o tempo de aleitamento. $O$ início da oferta das frutas e sopas apresentou mediana de 150 dias e da carne, 210 dias. Houve associação estatística entre a idade e o aumento da consistência. Verificou-se ainda, que os alimentos estão sendo ofertados em consistências adequadas para a idade. Conclusão: concluiuse que houve prática de aleitamento materno exclusivo com período menor ao que é recomendado. Os alimentos foram introduzidos precocemente, porém, a consistência está sendo adequada na alimentação atual. Pode-se verificar, também, a grande frequência do uso de mamadeira e chupeta como hábitos orais deletérios. Ainda, a orientação sobre a alimentação foi feita por pessoas leigas, na sua maioria.
\end{abstract}

DESCRITORES: Saúde Pública; Aleitamento Materno; Alimentação; Criança

\section{INTRODUÇÃO}

Os costumes alimentares são práticas sociais, pois nutrir-se, comer, são ações que ultrapassam o ato biológico. A nutrição de uma população não depende somente do acesso da mesma a uma alimentação adequada, mas, da educação e cul-

(1) Fonoaudióloga; Clínica Aline Carneiro.

(2) Fonoaudióloga; Professora Adjunta do Curso de Fonoaudiologia da Universidade Luterana do Brasil, ULBRA, Canoas, RS; Especialista em Motricidade Oral; Mestre em Saúde Coletiva pela Universidade Luterana do Brasil

(3) Fonoaudióloga; Professora Adjunta do Curso de Fonoaudiologia da Universidade Luterana do Brasil, ULBRA, Canoas, RS; Especialista em Motricidade Oral; Mestre em Medicina: Ciências Médicas pela Universidade Federal do Rio Grande do Sul.

Conflito de interesse: INEXISTENTE tura deste grupo ${ }^{1,2}$. Ações envolvendo a alimentação, e sua influência no desenvolvimento das funções orais, são de suma importância para um maior esclarecimento e conscientização da comunidade ${ }^{1,2}$. Conforme alguns autores ${ }^{1}$, os fonoaudiólogos devem conhecer os hábitos alimentares para adequar suas condutas à realidade da população, visando desenvolver um trabalho eficaz, baseandose em dados concretos e atuais.

Na face humana encontram-se órgãos que atuam em diferentes funções, que por sua vez mudam e amadurecem com o desenvolvimento do indivíduo ${ }^{3}$. Através da sucção, recém-nascidos humanos são alimentados, iniciando-se então, movimentos funcionais de lábio, mandíbula e língua. Quando o bebê é amamentado no seio materno, as estruturas envolvidas são estimuladas, favorecendo o cresci- 
mento ósseo e a superação do retrognatismo, iniciando-se então, a remodelação das articulações temporomandibulares ${ }^{3}$.

Enquanto o sistema estomatognático não está preparado para a função mastigatória, a sucção é a forma apropriada para o indivíduo obter o alimento. Mas, o prolongamento da sucção, através da mamadeira, leva a um aumento de hábitos de sucção não-nutritiva ${ }^{3}$. O leite materno apresenta os nutrientes necessários para uma alimentação saudável e exclusiva até o sexto mês de vida. A partir dessa idade devem ser introduzidos, na alimentação do bebê, os alimentos complementares até dois anos $^{4-6}$.

A Organização Pan-Americana de Saúde/Organização Mundial da Saúde ${ }^{7}$ analisou dados sobre aleitamento materno constatando que este ainda apresenta baixos índices de frequência, curta duração e, com sua prática exclusiva, raramente ocorrendo até os seis meses de vida. A mesma fonte concorda com autores que referem que há precoce proposta de alimentos complementares, com oferecimento, na maioria das vezes, de mamadeira muito frequente, durante o primeiro ano de vida, o que leva ao risco de desmame precoce ${ }^{8,9}$.

Assim que o pediatra liberar a dieta do bebê, devem ser oferecidos todos os tipos de alimentos (cereais, grãos, verduras, frutas, carnes, legumes), pois permitem conhecer e apreciar os mais variados tipos de sabores, consistências e texturas, desde cedo. A criança precisa mastigar para continuar tendo um bom desenvolvimento das suas estruturas orais ${ }^{7-9}$.

Com o decorrer do crescimento, a escolha e o preparo dos alimentos da dieta infantil representam a continuidade no fortalecimento dos músculos, crescimento e desenvolvimento das estruturas ósseas faciais, que serão responsáveis para a adequada realização das funções estomatognáticas ${ }^{3}$. Portanto, justifica-se que o fonoaudiólogo, assim como demais profissionais da saúde, deva observar e considerar o comportamento alimentar, para promover hábitos alimentares saudáveis que terão importância fundamental na prevenção de alterações miofuncionais ${ }^{1,2}$.

Sendo assim, o objetivo geral deste estudo foi caracterizar a alimentação de crianças de seis aos 24 meses. E especificamente, verificar a época de introdução, tipos de alimentos e texturas que estão sendo oferecidos, nesta faixa etária; constatar a presença de hábitos orais deletérios e levantar de quais profissionais ou fontes os pais receberam orientações sobre o processo de transição de texturas dos alimentos.

\section{MÉTODOS}

Realizou-se um estudo descritivo, quantitativo e transversal para caracterizar a alimentação de crianças de seis a 24 meses de idade, que frequentaram duas Unidades Básicas de Saúde (UBS), no município de Canoas-RS.

A amostra foi selecionada de forma não-probabilística, formada pelas primeiras 100 crianças atendidas em duas unidades básicas de saúde, no período de novembro de 2006 a março de 2007. Foi realizada uma entrevista com 100 responsáveis que freqüentaram a UBS SABETRAN e a UBS Estância Velha (50 responsáveis de cada Unidade Básica de Saúde), no município de Canoas. O critério de inclusão foi a idade de seis a 24 meses do bebê que compareceu à UBS. Foram excluídas da pesquisa as crianças com doenças neurológicas, síndromes, má formação de cabeça e pescoço e desnutrição.

Ao término das consultas pediátricas ou após procedimentos de vacinação ocorridos nos locais, os responsáveis eram convidados a participar do estudo. Depois de aceito o convite, a entrevista era realizada em uma sala disponibilizada pelas Unidades Básicas de Saúde. Na atual pesquisa foi realizado um estudo-piloto constituído de seis entrevistas (sendo três entrevistas de cada Unidade Básica de Saúde) com o objetivo de verificar a necessidade de possíveis ajustes na entrevista.

Esta pesquisa teve como instrumento de coleta uma entrevista realizada com os responsáveis pela criança com perguntas referentes ao parto, às condições ao nascimento, à alimentação da mesma até a presente data, com tipos e modos de preparo dos alimentos e utensílios utilizados para alimentação das crianças da amostra, assim como hábitos orais. A entrevista foi composta por 43 perguntas, sendo que duas destas eram questões abertas.

Esta pesquisa foi avaliada e aprovada pelo Comitê de Ética da Universidade Luterana do Brasil sob o protocolo número CEP-ULBRA 2005-3414 e os participantes da pesquisa assinaram um termo de consentimento, denominado Termo de Consentimento Livre e Esclarecido, onde se dispuseram a participar da investigação a que se propuseram os pesquisadores. Com isso, todos os participantes tiveram assegurados os direitos de sigilo, voluntariado e interrupção da participação, a qualquer tempo.

As informações do estudo foram armazenadas em um banco de dados no programa Excel e para análise estatística foi utilizado o programa SPSS/ PC 10.0., com uso de medidas de tendência central e tabelas cruzadas.

Para verificar possíveis associações entre as variáveis selecionadas da amostra, foram utilizados 
o teste Qui-quadrado e o teste Exato de Fischer (Fletcher e Wagner, 1996), considerando-se um nível de significância de 5\%.

\section{RESULTADOS}

A distribuição das crianças segundo o sexo foi de $60(60 \%)$ para masculino e $40(40 \%)$ para feminino, quanto à idade gestacional $94(94 \%)$ nasceram a termo e seis $(6 \%)$ pré-termo e $62(62 \%)$ da amostra de parto vaginal e 38 (38\%) cesárea. A idade média das crianças que participaram do estudo foi de um ano e dois meses.
Em relação às características dos responsáveis entrevistados, a idade média foi de 28 anos e 80 (80\%) deles apresentaram nível de instrução de ensino fundamental incompleto.

A prática do aleitamento materno exclusivo foi realizada por 98 (98\%) das crianças, porém a média de duração foi de apenas dois meses e oito dias.

Consequentemente, encontrou-se na presente pesquisa a introdução precoce de líquidos: o chá com mediana de início aos 60 dias de vida e a água com início de oferta aos 90 dias, conforme Tabela 1.

Tabela 1 - Distribuição da amostra conforme medianas (dias) da idade de introdução de líquidos

\begin{tabular}{lccc}
\hline Líquidos & Consumiam & Não consumiam & Mediana (Dias) \\
\hline Idade de introdução chá & $88 \%$ & $12 \%$ & 60 \\
Idade de introdução água & $100 \%$ & $0 \%$ & 90 \\
Idade de introdução de sucos & $93 \%$ & $7 \%$ & 150 \\
Idade de introdução & $75 \%$ & $25 \%$ & 270 \\
refrigerante & & & $25 \%$ \\
\hline
\end{tabular}

$\mathrm{n}=100$

Ao verificar a idade de introdução e a frequência do consumo de refrigerante na dieta das crianças pesquisadas, observou-se que das 100 crianças analisadas, 75 (75\%) consumiam esta bebida e 23 $(30,7 \%)$ delas apresentavam idade de início anterior ou igual a seis meses de idade (Tabela 2).

Tabela 2 - Distribuição da amostra conforme idade de introdução da bebida refrigerante

\begin{tabular}{lcc}
\hline Grupos etários & $\mathbf{n}$ & $\%$ \\
\hline 0 a 6 meses & 23 & 30,7 \\
7 a 12 meses & 41 & 54,7 \\
Acima de 12 meses & 11 & 14,6 \\
\hline Total & 75 & 100 \\
\hline
\end{tabular}

Quanto à duração da amamentação complementada, a atual pesquisa apontou mediana de 150 dias de vida e analisando o percentual das crianças que não se alimentavam mais em seio materno, identificou-se que $60(60 \%)$ da amostra total já haviam realizado desmame, com média de idade de cinco meses e 10 dias.

$\mathrm{Na}$ alimentação atual, de 92 (92\%) das crianças apenas oito $(8 \%)$ não faziam uso da mamadeira. Dos $92(92 \%)$ das crianças analisadas, em 47
(47\%) houve aumento do furo e em 64 (64\%) houve consumo tanto de líquidos engrossados como ralos, neste utensílio.

Em relação à frequência do uso da chupeta, verificou-se que $60(60 \%)$ faziam uso da mesma. $\mathrm{Na}$ atual pesquisa identificou-se, ainda, associação significante $(p=0,036)$ entre as crianças que receberam menor período de aleitamento materno exclusivo e o uso da chupeta (Tabela 3 ).

Nos dados apresentados na Tabela 4, verifica-se associação significante $(p=0,000)$ entre o aumento das faixas etárias e o oferecimento gradual das consistências mais sólidas para a sopa. A mediana de introdução foi de 150 dias, resultado igual ao verificado com as frutas que também apresentaram associação significativa $(p=0,000)$ entre o aumento da idade e texturas mais firmes.

A introdução da carne na população analisada revelou mediana de 210 dias, e também apresentou associação entre o aumento de idade e seu consumo $(p=0,011)$.

$\mathrm{Na}$ presente pesquisa ao questionar os responsáveis sobre as fontes de obtenção de informações para introdução dos alimentos e das consistências aos seus filhos, observou-se que a maior parte dos responsáveis citou pessoas conhecidas, sendo citado, em segundo lugar o pediatra, conforme Tabela 5. 
Tabela 3 - Distribuição da amostra conforme cruzamento uso da chupeta e tempo de amamentação exclusiva

\begin{tabular}{|c|c|c|c|c|}
\hline \multirow{2}{*}{ Tempo de amamentação } & & \multicolumn{2}{|c|}{ Uso de chupeta } & \multirow{2}{*}{ Total } \\
\hline & & Sim & Não & \\
\hline \multirow{2}{*}{0 a 60 dias } & $\mathrm{n}$ & 42 & 19 & 61 \\
\hline & $\%$ & $68,90 \%$ & $31,10 \%$ & $100,00 \%$ \\
\hline \multirow{2}{*}{61 a 120 dias } & $\mathrm{n}$ & 10 & 9 & 19 \\
\hline & $\%$ & $52,60 \%$ & $47,40 \%$ & $100,00 \%$ \\
\hline \multirow{2}{*}{121 a 180 dias } & $\mathrm{n}$ & 4 & 10 & 14 \\
\hline & $\%$ & $28,60 \%$ & $71,40 \%$ & $100,00 \%$ \\
\hline \multirow{2}{*}{181 a 240 dias } & $\mathrm{n}$ & 3 & 1 & 4 \\
\hline & $\%$ & $75,00 \%$ & $25,00 \%$ & $100,00 \%$ \\
\hline \multirow{2}{*}{ Total } & $\mathrm{n}$ & 59 & 39 & 98 \\
\hline & $\%$ & $60,20 \%$ & $39,80 \%$ & $100,00 \%$ \\
\hline
\end{tabular}

Teste Exato de Fischer $p=0,036$

Tabela 4 - Distribuição da amostra conforme cruzamento de grupos etários e consistência da sopa

\begin{tabular}{lccccc}
\hline Grupos etários & & Liquidificado & Amassado & Cortado & Total \\
\hline \multirow{2}{*}{ 6 a 12 meses } & $\mathrm{n}$ & 8 & 21 & 6 & 35 \\
& $\%$ & $22,90 \%$ & $60 \%$ & $17,10 \%$ & $100 \%$ \\
\multirow{2}{*}{13 a 18 meses } & $\mathrm{n}$ & 0 & 12 & 14 & 26 \\
\multirow{2}{*}{18 a 24 meses } & $\%$ & $0 \%$ & $46,20 \%$ & $53,80 \%$ & $100 \%$ \\
& $\mathrm{n}$ & 0 & 6 & 18 & 24 \\
\multirow{2}{*}{ Total } & $\%$ & $0 \%$ & $25 \%$ & $75 \%$ & $100 \%$ \\
\hline
\end{tabular}

Teste Exato de Fischer $p=0,000$

Tabela 5-Distribuição da amostra conforme relato dos responsáveis sobre fontes de informação quanto a aconselhamento no oferecimento das diferentes consistências dos alimentos

\begin{tabular}{lcc}
\hline Fonte de Informação & $\mathbf{n}$ & $\%$ \\
\hline Pessoas conhecidas & 80 & 80 \\
Pediatra & 48 & 48 \\
Nutricionista & 4 & 4 \\
Outros profissionais ou fontes & 5 & 5 \\
\hline
\end{tabular}

Resposta múltipla_Base: 100

\section{DISCUSSÃO}

A prática do aleitamento materno exclusivo foi realizada por $98 \%$ das crianças, porém a média de duração foi de apenas dois meses e oito dias. Estes dados demonstram estar distantes do recomendado pela Organização Pan-Americana de Saúde/Organização Mundial da Saúde ${ }^{7}$, que orienta o consumo exclusivo de leite materno até o $6^{\circ}$ mês de vida.

Autores brasileiros ${ }^{10}$, ao pesquisarem a prevalência do aleitamento materno exclusivo, verificaram que $97,9 \%$ das crianças foram amamentadas exclusivamente, apresentando duração inferior ao atual estudo, com média de um mês e cinquenta e um dias. Já em outro estudo ${ }^{11}$ ao analisarem o padrão de aleitamento em outra amostra, observaram que apenas $39 \%$ dos bebês da amostra recebiam o leite materno exclusivo aos três meses de idade, portanto, idade bem inferior ao preconizado.

Analisando as crianças que não se alimentavam mais ao seio materno, identificou-se que $60 \%$ da amostra total já haviam realizado desmame com média de idade de cinco meses e 10 dias.

Outra pesquisa ${ }^{12}$ constatou a ocorrência de desmame em período mais precoce que o encontrado no atual estudo: a maior parte de sua amostra desmamou por volta do terceiro mês de vida. Dado 
também encontrado em estudo em São Paulo ${ }^{13}$, no qual, em média, aos três meses de idade $57,8 \%$ da amostra apresentava o processo de desmame.

Em pesquisa com crianças de 6 aos 12 meses de idade, realizada no município de Canoas ${ }^{14}$, o desmame apresentou ocorrência mais precoce que o presente estudo, com média de 77 dias (dois meses e 17 dias).

O chá e a água apresentaram medianas de início de oferta aos 60 e 90 dias respectivamente, determinando o que se considera como precocidade de oferta de líquidos, achado que concorda com a literatura consultada. Autores ${ }^{4}$ verificaram em seu estudo sobre hábitos alimentares maior consumo de chá comparado à água e ao suco em crianças menores de um ano de idade, atribuindo este resultado a fatores culturais ligados ao seu uso como medicação e não como nutrição. Outros pesquisadores ${ }^{11}$ encontraram dados semelhantes, uma vez que $13 \%$ dos bebês da amostra do estudo realizavam consumo de chá aos três meses de vida, com responsáveis atribuindo a introdução precoce deste líquido à presença de cólicas, gases ou sede. Resultados no mesmo sentido foram obtidos em outra ampla pesquisa ${ }^{10}$, os quais mostraram que $33,6 \%$ das crianças já recebiam chás e $12,4 \%$ consumiam água, com menos de um mês de vida.

Ainda foi constatado em outro estudo ${ }^{4}$ que o consumo de suco e água pelas crianças amamentadas interfere menos do que o chá nos indicadores de desmame precoce, sinalizando a necessidade de medidas de intervenção através de um trabalho educativo com a população. Sabe-se que mudar hábitos culturalmente estabelecidos é difícil, e é necessário um trabalho contínuo, que respeite as características da comunidade.

Ao verificar a idade de introdução e a frequência do consumo de refrigerante na dieta das crianças pesquisadas, constatou-se que $75 \%$ consumiam esta bebida e $23(30,7 \%)$ delas apresentavam idade de início igual ou anterior a seis meses de idade. Cabe ressaltar diante destes dados, a recomendação da Organização Pan-Americana de Saúde/OMS ${ }^{7}$, a qual aborda 10 passos para prática de uma boa alimentação da criança até os dois anos de idade. $O$ passo número 8 refere-se a evitar o consumo de refrigerantes devido ao fato de não serem considerados bons para a nutrição, pois competem com alimentos nutritivos. Além disso, o açúcar deve ser oferecido com moderação nos primeiros anos de vida, de preferência, sendo introduzido a partir do $1^{\circ}$ ano de idade. Portanto, esta prática mostra-se inadequada na presente amostra pesquisada.

Com respeito ao uso da mamadeira, considerado um hábito oral deletério, $92 \%$ das crianças fizeram uso e $47 \%$ alargaram o furo do bico da mesma. Pesquisadoras do mesmo município ${ }^{14}$ ao analisarem a frequência da utilização da mamadeira, verificaram percentual aproximado ao encontrado neste estudo, ou seja, $89,5 \%$ faziam uso deste utensílio.

Estes dados revelam a importância do exposto pela literatura ${ }^{15}$, que alerta sobre o uso da mamadeira e os furos impróprios, muitas vezes aumentados pelos responsáveis, ocasionando movimentos incorretos de lábios e de toda a musculatura facial devido à falta de esforço na obtenção do leite. Vários autores ${ }^{9,16}$ concordam com a afirmação e atribuem este fato à falta de informação das mães. Acrescentam ainda que ao facilitar a sucção poderão ocorrer má formação de arcadas dentárias, flacidez da musculatura oral e facilidade na ocorrência de engasgos.

A alta frequência e o uso precoce da chupeta é uma tendência comum observada em estudos que abordam o comportamento infantil e seu impacto no aleitamento materno. Isto nos revela o quanto este hábito está inserido em nossa sociedade.

$\mathrm{Na}$ presente pesquisa constatou-se o uso em $60 \%$ da amostra e precocidade na introdução da chupeta com mediana de três dias. Resultado que concorda com o estudo ${ }^{17}$, realizado na cidade de Pelotas/RS, ao verificar que $62 \%$ da sua amostra iniciaram este hábito já no primeiro dia de vida.

$\mathrm{Na}$ atual pesquisa identificou-se ainda associação significativa $(p=0,036)$ entre as crianças que receberam menor período de aleitamento materno exclusivo e uso da chupeta. Outros autores ${ }^{18}$ também constataram que o uso da chupeta foi mais frequente entre as crianças que não estavam sendo amamentadas exclusivamente no primeiro mês, $73,1 \%$ das que usavam chupeta deixaram de ser amamentadas exclusivamente até o final do segundo mês.

$\mathrm{Na}$ introdução das sopas, o presente estudo verificou mediana igual à apresentada pelas frutas, 150 dias. Em estudo semelhante sobre hábitos alimentares ${ }^{10}$ apresentados por crianças até os 24 meses de idade constataram introdução mais cedo, com sopas sendo oferecidas por volta dos 4 meses (120 dias).

Verificou-se ainda uma associação significativa entre as variáveis, idade da criança e a consistência de frutas e de sopa, $(p=0,000)$, no presente estudo, dado que concorda com pesquisa ${ }^{16}$ que constatou a introdução precoce de frutas, com ascensão e constante consumo nas faixas etárias dos três aos nove meses de idade.

Estes dados eram esperados uma vez que com o decorrer da idade as crianças desenvolvem estruturas orofaciais que possibilitam a mastigação de consistências mais sólidas. Já por volta do $6^{\circ}$ mês 
de idade, a sopa de legumes deve ser oferecida com consistência já espessa ${ }^{8}$. A literatura ${ }^{19}$ indica que os novos alimentos salgados devem ser passados na peneira e não liquidificados, pois a consistência muito lisa pode retardar o processo da mastigação. Além disso, o alimento peneirado propicia movimentos laterais da língua em busca dos alimentos espalhados na cavidade oral.

No oitavo mês, a sopa deve ser servida amassada com o garfo para desenvolver melhor a musculatura facial e a função mastigação, e entre o $9^{\circ} \mathrm{e}$ o $12^{\circ}$ mês, ela já pode ser ofertada com os legumes em pedaços cozidos ou levemente amassados ${ }^{8}$.

A introdução da carne na amostra analisada revelou mediana de 210 dias, resultado mais tardio quando comparado ao resultado encontrado em outra pesquisa semelhante ${ }^{19}$, onde a mediana apresentou 180 dias para início da oferta deste alimento. Durante o $7^{\circ}$ mês e o primeiro ano de vida, refere-se que a carne moída ou desfiada já pode ser ofertada, pois a alimentação da criança começa a ter característica mais sólida ${ }^{8}$, o que concorda com os dados levantados na atual pesquisa, na qual houve associação estatística entre o aumento da idade e o consumo de carne em pedaços.

$\mathrm{Na}$ presente pesquisa ao questionar os responsáveis sobre as fontes de obtenção de informações para introdução dos alimentos e das consistências aos seus filhos, observou-se que a maior parte citou pessoas conhecidas e em segundo lugar, um profissional da saúde, o pediatra. Pesquisa realizada em Recife ${ }^{12}$ constatou a participação de leigos como maiores instrutores em relação ao oferecimento de orientações da dieta alimentar e de higiene bucal em sua amostra, sendo em segundo lugar também citado o pediatra, mais que outros profissionais da saúde, dado que concorda com o estudo realizado.

Em vista desses resultados, os autores são unânimes ao apontar a importância do planejamento de ações e programas de caráter educativo dentro do campo da Saúde Coletiva para a prática do aleitamento materno exclusivo e a promoção de estratégias que visam orientar e aconselhar os responsáveis sobre a introdução da alimentação complementar infantil 10,11,13,20. Mais pesquisas nesse sentido deveriam ser realizadas, inclusive por fonoaudiólogos.

O campo da Saúde Coletiva abre novas perspectivas para os fonoaudiólogos no sentido de assumir o nosso lugar social como sujeitos da ação para uma nova prática de relações com os outros campos da saúde e com a comunidade. Resultados de pesquisas como as que aqui foram discutidas demonstram a necessidade de participar mais efetivamente na promoção de hábitos alimentares saudáveis que terão importância fundamental na prevenção de alterações do desenvolvimento infantil ${ }^{21}$.

\section{CONCLUSÃO}

Constatou-se com este estudo, que o aleitamento materno exclusivo foi pouco praticado até os seis meses, conseqüentemente, houve a introdução precoce de líquidos e pastosos na dieta das crianças. A amamentação complementada também apresentou baixos índices de duração demonstrando resultados distantes do recomendado pela OMS. A iniciação do consumo do refrigerante, que não deveria estar presente na dieta alimentar da população estudada, apresentou-se em período precoce da vida. Nas variáveis que abordavam o preparo dos alimentos, constatou-se que a consistência, da maior parte das crianças, estava sendo ofertada de forma adequada atualmente. Quanto à presença de hábitos orais deletérios, os dados mostraram que praticamente a totalidade da população pesquisada faz uso muito freqüente da chupeta e da mamadeira. E, a partir dos dados coletados, identificou-se uma grande participação de leigos nas orientações aos responsáveis sobre a introdução dos alimentos e texturas a serem ofertadas. 


\section{ABSTRACT}

Purpose: to characterize the feeding in children aged from 6 to 24-month old; and specifically, to characterize the time of introduction, types of foods and textures offered according to child's age, to check oral habits and to verify who guided the parents about feeding. Methods: the sample was composed by 100 children of 6 to 24 months, which attended two Basic Health Care Units, in Canoas, RS. Interviews were accomplished with the sponsors. Results: it was verified that $98(98 \%)$ of the children were exclusively breastfeed, however with medium duration of 2 months and 8 days. The offer of liquids presented a precocious introduction of tea at the beginning with median of 60 days; 92 (92\%) of the children used bottle-feeding and in $47(47 \%)$ of them increased the hole. The pacifier was used by $60(60 \%)$ of the sample and had an association with the time of breast feeding. The beginning of offering fruits and soups presented a median of 150 days and meat in 210 days. There was a statistical association between the age and increase of consistence. It was still verified, that foods are being presented in appropriate consistence for the age. Conclusion: it was concluded that there was an exclusive practice of maternal breastfeeding with period shorter than it is recommended. Foods were introduced before the proper time; however, the consistence is being adapted in the current feeding. It can also be verified the great frequency for employing bottle-feeding and pacifier as a deleterious oral habits. Most of the time, orientation about feeding was made by lay people.

KEYWORDS: Public Health; Breast Feeding; Feeding; Child

\section{REFERÊNCIAS}

1. Medeiros JF, Maciel CGB, Motta, AR. Levantamento dos hábitos alimentares de crianças de 4 a 6 anos: base para um trabalho preventivocomunitário. Rev CEFAC 2005; 7(2):198-204.

2. Rotenberg S, De Vargas S. Práticas alimentares e o cuidado da saúde: da alimentação da criança à alimentação da família. Rev Bras Saúde Matern Infant. 2004; 4(1):85-94.

3. Felício CM. Sistema estomatognático e funções. In: Felício CM. Fonoaudiologia aplicada a casos odontológicos: motricidade oral e audiologia. São Paulo: Pancast; 1999. p. 15-35.

4. Vieira GO, Silva LR, Vieira TO, Almeida JAG, Cabral VA. Hábitos alimentares de crianças menores de 1 ano amamentadas e não-amamentadas. J Pediatr. 2004; 80(5):411-6.

5. Monte CMG, Giugliani ERJ. Recomendações para alimentação complementar da criança em aleitamento materno. J Pediatr. 2004; 80(Supl5): 131-41.

6. Spinelli MGN, Souza SB, Souza JMP. Mamadeira, xícara ou colher: de que forma os bebes estão recebendo os alimentos?. Pediatr Moder. 2002; 38(10):461-8.

7. Brasil. Ministério da Saúde/Organização PanAmericana da Saúde. Bases Científicas atuais da alimentação da criança menor de dois anos. In: Guia alimentar para crianças menores de 2 anos de idade. Série A. Normas e manuais técnicos. Brasília, DF: Ministério da Saúde; 2002. p.15-23.
8. Mello MMS. Aleitamento materno e a evolução da alimentação infantil. In: Mello MMS. Educação e nutrição: uma receita de saúde. Porto Alegre: Mediação; 2003. p. 25-49.

9. Junqueira $P$. Amamentação, hábitos orais e mastigação: orientações, cuidados e dicas. Rio de Janeiro: Revinter; 1999. p. 1-25.

10. Silveira FJF, Lamounier JA. Prevalência do aleitamento materno e práticas de alimentação complementar em crianças com até 24 meses de idade na região de Alto Jequitinhonha, Minas Gerais. Rev Nutr. 2004; 17(4):437-47.

11. Mascarenhas MLW, Albernaz EP, Silva MB, Silveira RB. Prevalência de aleitamento materno exclusivo nos 3 primeiros meses de vida e seus determinantes no Sul do Brasil. J Pediatr. 2006; 82(4):289-94.

12. Alves TDB, Montandon EM, Menezes VA. Levantamento epidemiológico em crianças de 0 a 30 meses na cidade de Recife-PE. Parte I: avaliação da dieta e higiene bucal. Rev Odontol Bras Central. 1998; 7(23):44-9.

13. Souza SB, Szarfarc SC, Souza JMP. Prática alimentar no primeiro ano de vida, em crianças atendidas em centros de saúde escola do município de São Paulo. Rev Nutr. 1999; 12(2):167-74.

14. Pereira LSN, Delgado SE. Caracterização do desenvolvimento da alimentação em crianças de 6 a 12 meses atendidas na UBS - CAIC. [monografia] Canoas (RS): Universidade Luterana do Brasil; 2006. 
15. Cunha VLO. Prevenindo problemas na fala pelo uso adequado das funções orais: Manual de orientação. São Paulo: Pró-Fono; 2001. p. 3-39.

16. Brunken GS, Silva SM, França GVA, Escuder MM, Venâncio SI. Fatores associados à interrupção precoce do aleitamento materno exclusivo e à introdução tardia da alimentação complementar no centro-oeste brasileiro. J Pediatr. 2006; 82(6):445-51.

17. Tomassi E, Victora CG, Olinto MTA. Padrões e determinantes do uso de chupeta em crianças. J Pediatr. 1994; 70(3):167-71.

18. Soares MEM, Giugliani ER, Braun ML, Salgado ACN, Oliveira AP, Aguiar PR. Uso de chupeta e sua relação com o desmame precoce em população de crianças nascidas em Hospital Amigo da Criança. J Pediatr. 2003; 79(4):309-16.

19. Simon VGN, Souza JMP, Souza SB. Introdução de alimentos complementares e sua relação com variáveis demográficas e socioeconômicas, em crianças no primeiro ano de vida, nascidas em Hospital Universitário no município de São Paulo. Rev Bras Epidemiol. 2003; 6(1):29-38.

20. Saldiva SRDM, Escuder MM, Mondini L, Levy RB, Venancio SI. Práticas alimentares de crianças de 6 a 12 meses e fatores maternos associados. J Pediatr. 2007; 83(1):53-8.

21. Delgado SE. A fonoaudiologia no contexto da promoção de saúde coletiva materno-infantil. Fono Atual. 2004; 7(29):72-5.

RECEBIDO EM: 29/06/2008

ACEITO EM: 15/01/2009

Endereço para correspondência:

Susana Elena Delgado

Rua Pedro Chaves Barcellos, 892/201

Porto Alegre - RS

CEP: 90450-010

E-mail: sudel.ez@ terra.com.br 